\title{
8. SEQUENTIAL AND SYNCHRONOUS SKIN PATTERN FORMATION
}

\author{
Gerhard C. Cruywagen ${ }^{1}$, P. K. Maini ${ }^{2}$ and J. D. Murray ${ }^{1}$ \\ ${ }^{1}$ Department of Applied Mathematics FS-20 \\ University of Washington \\ Seattle, WA 98195, USA \\ ${ }^{2}$ Centre for Mathematical Biology \\ Mathematical Institute \\ 24-29 St. Giles' \\ Oxford OX1 3LB, UK
}

\subsection{INTRODUCTION}

Mathematical modelling has become a widely accepted method for examining how and why vertebrate skin structures are laid down in an orderly and organized fashion. Although various theoretical models have been proposed for examining the morphogenetic processes responsible for the large variety of patterns observed on animal skin, these processes are still not well understood. By examining a mechanochemical tissue interaction model based on recent experimental evidence we therefore hope to contribute towards the understanding of skin morphogenesis.

Vertebrate skin is composed of two layers: the epidermis, made up of sheets of columnar cells, overlies the dermis, consisting of motile cells which move about on the extracellular matrix (ECM). These two layers are separated by the fibrous basal lamina. The first stages of appendage formation is characterized by dermal cell aggregation centres, the papillae, which form directly underneath and simultaneously with epidermal thickenings, the placodes. Experimental evidence (for example, Gallin et al. 1986; Chuong \& Edelman 1985) indicates that interaction between the epithelial and dermal layers plays an important role during skin appendage formation.

Apart from the model of Cruywagen \& Murray (1992), all the theoretical tissue interaction mechanisms proposed so far involve reaction-diffusion systems (see for example, Nagorcka 1986; Shaw \& Murray 1990). For a brief review on the modelling of tissue interaction refer to Murray et al. (1993). There is, however, still little experimental evidence for the existence of such reacting and diffusing morphogens in skin pattern formation. Therefore we examine here the two-dimensional synchronous and sequential pattern formation capabilities of Cruywagen \& Murray's (1992) mechanochemical model. 


\subsection{THE TISSUE INTERACTION MODEL}

The model of Cruywagen \& Murray (1992) is a continuum tissue interaction model and consists of two parts: an equation to describe dermal cell movement and two equations for describing epithelial sheet deformation. These two sub-models are coupled by introducing tissue interaction. We briefly describe this model here and refer the reader to the original paper for full details.

The epithelial sheet is modelled as a two-dimensional, visco-elastic continuum (see for example, Murray \& Oster 1984; Murray 1989). As the system is in a low Reynold's number regime, we assume that the visco-elastic and cell traction stresses within the epidermis are balanced by the external body forces. The force balance equation takes the form

$$
\begin{gathered}
\nabla \cdot\{\overbrace{\frac{E}{1+v}\left[\varepsilon-\beta_{1} \nabla^{2} \varepsilon+\frac{v}{1-2 v}\left(\theta-\beta_{2} \nabla^{2} \theta\right) \boldsymbol{I}\right]}^{\text {elastic stress }} \\
+\overbrace{\mu_{1} \frac{\partial \varepsilon}{\partial t}+\mu_{2} \frac{\partial \theta}{\partial t} \boldsymbol{I}}^{\text {viscous stress }}+\overbrace{\tau(s) \boldsymbol{I}}^{\text {traction }}\}=\overbrace{\rho \boldsymbol{u}}^{\text {body forces }},
\end{gathered}
$$

where the variable $\boldsymbol{u}(\boldsymbol{x}, t)$ is the displacement at time $t$ of a material point in the epithelial layer which was initially at position $\boldsymbol{x}, \boldsymbol{\varepsilon}=\left(\nabla \boldsymbol{u}+\nabla \boldsymbol{u}^{T}\right) / 2$ is the strain tensor, $\theta=\nabla \cdot \boldsymbol{u}$ the dilation, $T$ denotes the transpose, and $s$ is the concentration of a signalling chemical. The parameter $E$ is Young's modulus, $v$ is Poisson's ratio, $\mu_{1}$ and $\mu_{2}$ are the shear and bulk viscosities, respectively (Landau \& Lifshitz 1970), and $I$ is the unit tensor. The parameters $\beta_{1}$ and $\beta_{2}$ measure the long range elastic stresses (see Murray 1989 for a discussion). The epidermis is attached to the basal lamina with adhesion tethers; $\rho$ reflects the strength of these attachments. The epithelial sheet exerts active traction which we assume depends on the signal chemical $s$, which diffuses from the dermis into the epidermis, thus introducing tissue interaction. We model this traction as the switch, $\tau(s)=\tau s^{2}(n) /\left(1+c s^{2}(n)\right)$, where $\tau$ and $c$ are positive constants (see Murray \& Oster 1984).

An epithelial cell conservation equation relates the epidermal cell density $N(x, t)$ to the displacement $\boldsymbol{u}$. Since the only contribution to cell flux is convection, the equation is simply

$$
\frac{\partial N}{\partial t}=\overbrace{-\nabla \cdot N \frac{\partial \boldsymbol{u}}{\partial t}}^{\text {convection }} .
$$

To model dermal morphogenesis we consider a chemotaxis equation, related to the cellchemotaxis model of (Oster \& Murray 1989) and based on the Morphoregulator Hypothesis of Edelman (see for example, Edelman 1986). According to this hypothesis skin organ morphogenesis is controlled by cell-cell adhesion mechanisms mediated by cell adhesion molecules (CAMs). Because chemical modulation can have a marked effect on the binding rates and binding strengths of CAMs (Grumet \& Edelman 1988), we assume that a chemical signal concentration $e$, diffusing from the epidermis into the dermis, is responsible for CAM expression. The conservation equation for dermal cell density, $n(\boldsymbol{x}, t)$, then takes the form

$$
\frac{\partial n}{\partial t}=\overbrace{\nabla \cdot D \nabla n}^{\text {diffusion }}-\overbrace{\nabla \cdot n \nabla \alpha(e)}^{\text {chemotaxis }}+\overbrace{r n\left(n_{0}-n\right)}^{\text {mitosis }},
$$


where $D$ is the coefficient of random diffusion and where we have assumed that cell growth obeys the logistic law, with $r$ and $n_{0}$ positive constants. It is assumed that the function $\alpha(e)$ models the chemo-attraction and that it has the linear form $\alpha(e)=\alpha e(N)$, where $\alpha$ is a non-negative constant and $e$ is a function of $N$ (see Cruywagen \& Murray 1992).

The system (8.1), (8.2) and (8.3) constitutes the field equations of our tissue interaction model. The full system is extremely complex, but by making a few reasonable biological assumptions, (Cruywagen \& Murray 1992) reduced it to two coupled nonlinear equations, thus making it more amenable to analysis while still retaining the essential biological features of the full model.

They obtained this simpler version by taking the divergence of the epidermal tensor equation (8.1), thus reducing it to a scalar dilation equation in $\theta$. They also applied the small strain assumption to equation (8.2) to obtain a linear relationship between epidermal cell density and dilation. The reduced model involves only the epithelial dilation $\theta$ and the dermal cell density, $n$.

\subsection{SYNCHRONOUS AND SEQUENTIAL PATTERN FORMATION}

Initially we examined the synchronous pattern formation capabilities of Cruywagen \& Murray's (1992) reduced model. We solved the reduced system numerically on rectangular domains using zero-flux boundary conditions in cell density and dilation. As initial conditions small random perturbations about the homogeneous steady state were specified. Parameter values were chosen so that the linearized version of the problem had only one unstable eigenvalue satisfying the boundary conditions. This eigenvalue could, for example, be a simple or a multiple eigenvalue (see Cruywagen et al. 1993 for an explanation). The model can exhibit several different types of patterned solutions such as squares, rhombi, rolls, hexagonal and mixed mode solutions.

However, in many developmental situations spatial pattern formation occurs sequentially. Examples of such sequential patterning include chick feather germ initiation (see for example, Chuong \& Edelman 1985) and alligator skin pigmentation (Murray et al. 1990). (Nagorcka 1986) examined the sequential pattern formation capabilities of reactiondiffusion systems on two-dimensional domains. Here we apply the tissue interaction model described in Section 2 to sequential pattern formation.

Numerical simulations of the model revealed that the spatial pattern which propagates across the domain depends crucially on the pattern that forms initially at one end of the domain. Figure 8.1 shows that if spots are initially specified at one end the resultant propagating pattern is rhombic. On the other hand, if the initial pattern is stripes, then the resultant two-dimensional pattern would be stripes. A detailed discussion of the sequential aspects of pattern formation is presented in (Cruywagen et al. 1993).

ACKNOWLEDGMENTS GCC would like to thank The Rhodes Trust, Oxford, and the South African Foundation for Research Development for their financial support. This work (JDM) was in part supported by a Grant DMS-9106848 from the U. S. National Science Foundation. 

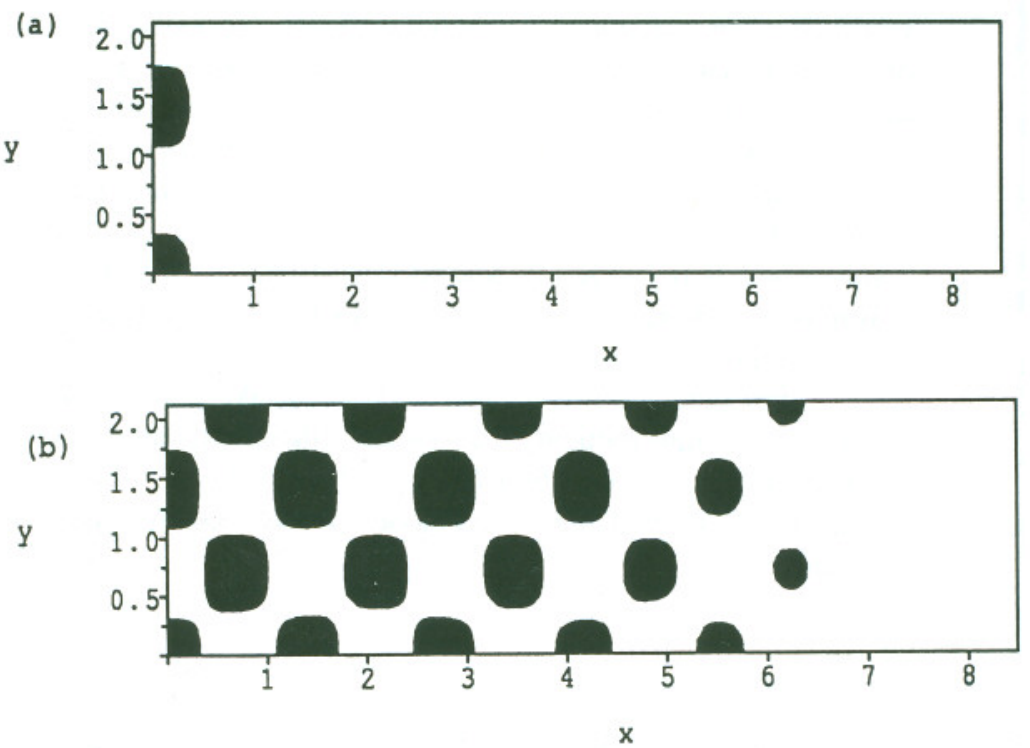

Figure 8.1. Numerical solutions of the caricature tissue interaction model. An initial row of spots (a) propagates across the domain to form a rhombic pattern (b).

\section{REFERENCES}

Chuong, C.-M., \& Edelman, G. M. 1985. Expression of cell adhesion molecules in embryonic induction. I. Morphogenesis of nestling feathers. J. Cell Biol., 101, 1009-1026.

Cruywagen, G. C., \& Murray, J. D. 1992. On a tissue interaction model for skin pattern formation. $J$. Nonlinear Sci., 2, 217-240.

Cruywagen, G. C., Maini, P. K., \& Murray, J. D. 1993. Sequential pattern formation in a model for skin morphogenesis. IMA J. Maths. Appl. Med. \& Biol. (In press).

Edelman, G. M. 1986. Cell adhesion molecules in the regulation of animal form and tissue pattern. Annu. Rev. Cell Biol., 2, 81-116.

Gallin, W. J., Chuong, C.-M., Finkel, L. H., \& Edelman, G. M. 1986. Antibodies to liver cell adhesion molecules perturb inductive interactions and alter feather pattern and structure. Proc. Natl. Acad. Sci. USA, 83, 8235-8239.

Grumet, M., \& Edelman, G. M. 1988. Neuron-glia cell adhesion molecules interact with neurons and astroglia via different binding mechanisms. J. Cell Biol., 106, 487-503.

Landau, L. D., \& Lifshitz, E. M. 1970. Theory of Elasticity. 2nd edn. New York: Pergamon.

Murray, J. D. 1989. Mathematical Biology. New York: Springer-Verlag.

Murray, J. D., \& Oster, G. F. 1984. Generation of biological pattern and form. IMA J. Maths Appl. Med. \& Biol, 1, 51-75.

Murray, J. D., Deeming, D. C., \& Ferguson, M. W. J. 1990. Size dependent pigmentation pattern formation in embryos of Alligator Mississipiensis: time of initiation of pattern generation mechanism. Proc. Roy. Soc., B239, 279-293.

Murray, J. D., Cruywagen, G. C., \& Maini, P. K. 1993. Pattern formation in tissue interaction systems. Heidelberg: Springer-Verlag. (In press) Lect. Notes in Biomathematics 100.

Nagorcka, B. N. 1986. The role of a reaction-diffusion system in the initiation of skin organ primordia. I. The first wave of initiation. J. Theor. Biol., 121, 449-475.

Oster, G. F., \& Murray, J. D. 1989. Pattern formation models and developmental constraints. J. exp. Zool., 251, 186-202.

Shaw, L. J., \& Murray, J. D. 1990. Analysis of a model for complex skin patterns. SIAM J. Appl. Math., 50(2), 628-648. 